\title{
Betti numbers of Stanley-Reisner rings determine hierarchical Markov degrees
}

\author{
Sonja Petrović · Erik Stokes
}

Received: 3 November 2011 / Accepted: 5 June 2012 / Published online: 28 June 2012

(C) Springer Science+Business Media, LLC 2012

\begin{abstract}
There are two seemingly unrelated ideals associated with a simplicial complex $\Delta$ : one is the Stanley-Reisner ideal $I_{\Delta}$, the monomial ideal generated by minimal non-faces of $\Delta$, well-known in combinatorial commutative algebra; the other is the toric ideal $I_{M(\Delta)}$ of the facet subring of $\Delta$, whose generators give a Markov basis for the hierarchical model defined by $\Delta$, playing a prominent role in algebraic statistics.

In this note we show that the complexity of the generators of $I_{M(\Delta)}$ is determined by the Betti numbers of $I_{\Delta}$. The unexpected connection between the syzygies of the Stanley-Reisner ideal and degrees of minimal generators of the toric ideal provide a framework for further exploration of the connection between the model and its many relatives in algebra and combinatorics.
\end{abstract}

Keywords Stanley-Resiner ideal · Betti numbers · Markov basis · Markov width · Toric ideal generators

\section{Introduction}

A central problem in algebraic statistics is the study of the combinatorial properties and complexity of Markov bases for toric models. In statistics, Markov bases provide an alternative, non-asymptotic approach to performing goodness-of-fit tests and

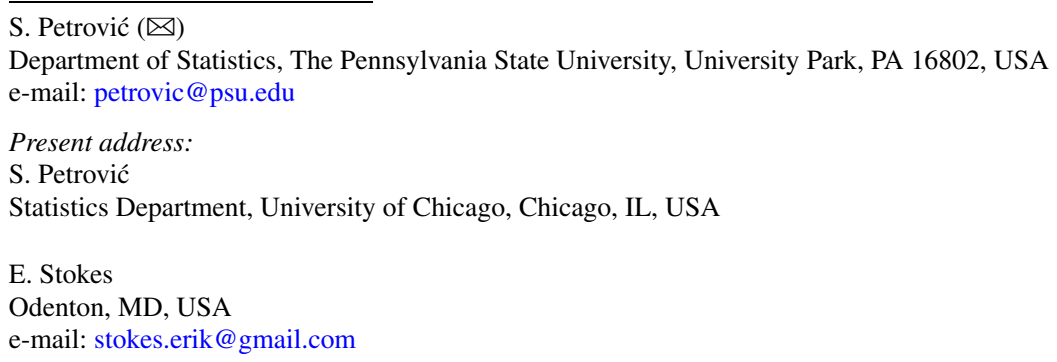


model selection. Their use has increased in recent years, especially for the models where the standard tools do not scale well. A statistical model is called algebraic if its parameter space is a semi-algebraic set. In this case, the model corresponds to the real positive part of the algebraic variety obtained by taking the Zariski closure of the image of the model parametrization map. When the variety is toric, any generating set of its defining ideal is a Markov basis for the model; this is a fundamental theorem that appeared in the breakthrough paper [7]. From a large and growing literature in algebraic statistics, we single out three recent books that can serve as an overview: [11, 24, 25].

Simplicial complexes define an important class of toric models called hierarchical models (defined in [13] and see also [19]). In a hierarchical model, relationships between $m$ discrete random variables are described by a simplicial complex $\Delta$ on $m$ vertices: the facets of $\Delta$ determine which margins of the corresponding $m$-way contingency table, or tensor, serve as minimal sufficient statistics for the model. The matrix $M(\Delta)$ of the sufficient statistics facet-margin linear map defines a toric ideal $I_{M(\Delta)}$ (see Sect. 2 for a precise definition).

A general open problem is to better understand how combinatorial (and other) properties of the simplicial complex determine the properties of the toric ideal $I_{M(\Delta)}$ of the hierarchical model. A crucial question is to determine the combinatorial complexity and degree estimates for the generators of the toric ideal. This problem has been studied for several families of models, but remains open in general. In algebraic statistics, the largest degree of a minimal generator of $I_{M(\Delta)}$ is called the Markov width of the model, as it provides the bound on the complexity of the moves needed for the Markov Chain used to walk on the space of observations. There are several interesting results in this direction, giving upper and lower bounds and solving this problem for special cases (for example [6] and [19], and for related work, see [2, 18]). In addition, if $\Delta$ is a reducible complex, then $I_{M(\Delta)}$ is a toric fiber product, so it is possible to lift the generators inductively (for the most general construction that applies to reducible complexes, see [27] and [12]).

The Stanley-Reisner ideal is a well-studied ideal associated to a simplicial complex $\Delta$ on $m$ vertices; it is the squarefree monomial ideal $I_{\Delta} \subseteq K\left[x_{1}, \ldots, x_{m}\right]$ generated by the minimal non-faces of $\Delta$. Betti numbers of Stanley-Reisner ideals have been studied by many authors from different points of view. Our study uses some known results and combinatorial techniques to provide a first link between Betti numbers of $I_{\Delta}$ and the degrees of minimal generators of $I_{M(\Delta)}$.

Our main result shows that the syzygies of the Stanley-Reisner ideal predict degrees of minimal generators of the toric ideal of the hierarchical model:

Theorem (Theorem 4.4) Let $\Delta$ be any simplicial complex. Suppose that the Betti diagram of the minimal free resolution of $R / I_{\Delta}$ has a non-zero entry in the $j$ th row (that is, $\beta_{i, i+j}\left(R / I_{\Delta}\right) \neq 0$ for some $\left.i\right)$. Then the toric ideal of the hierarchical model, $I_{M}$, has a minimal generator with degree $2^{j}$.

The proof of Theorem 4.4 is in Sect. 4, which also contains a geometric interpretation of the result. In Sect. 5 we outline a way to construct some of the predicted Markov moves from Theorem 4.4. We also carry out a computational summary of unpredictable moves in Sect. 6. 
One important family of complexes that arises in the theory of graphical models is decomposable complexes. For a detailed study, see for example [8] and [9], where they appear under the name decomposable graphical models. They correspond to the hierarchical models of clique complexes of chordal graphs (cf. Theorem 3.3.3 in [11]). In Corollary 4.10, we recover a result of Fröberg on linear resolutions for decomposable complexes.

The Betti diagram of $I_{\Delta}$ can vary with the ground field for general $\Delta$. In particular, the regularity may change. Recall that the regularity is defined to be the largest $j$ so that $\beta_{i, i+j}\left(R / I_{\Delta}\right) \neq 0$, that is, the number of rows in the Betti diagram. For example, the Alexander dual of any triangulation of the projective plane changes regularity when the field has characteristic 2. Our results hold over any field. Therefore, we can choose a "worst-case" field, where the regularity is largest, thus giving us most information about the Markov complexity of the hierarchical model.

The insight provided by the Betti numbers of the Stanley-Reisner ideal motivates interpretation of other classical numerical invariants of the coordinate ring. In terms of the model, these questions are still unexplored (Sect. 6 contains a few open problems). We hope this relationship will inspire a further study of the effect of the algebraic and geometric invariants of the classical combinatorial object on the underlying algebraic statistical model.

Before providing technical details, we illustrate the main Theorem on an example.

Example 1.1 Consider the binary model of a 4-cycle $\Delta$ with facets $\{12\},\{23\},\{34\}$, $\{14\}$. The Stanley-Reisner ideal $\left(x_{1} x_{3}, x_{2} x_{4}\right)$ has the following Betti diagram:

$\begin{array}{rlll} & 0 & 1 & 2 \\ \text { total: } & 1 & 2 & 1 \\ 0: & 1 & . & . \\ 1: & . & 2 & . \\ 2: & . & . & 1\end{array}$

Since rows 1 and 2 have non-zero entries, Theorem 4.4 states that the toric ideal $I_{M}$ must have generators in degrees $2^{1}=2$ and $2^{2}=4$.

The toric ideal $I_{M}$ lives in the polynomial ring with 16 variables $p_{i_{1} i_{2} i_{3} i_{4}}, i_{j} \in$ $\{0,1\}$, which refer to the binary states of four random variables corresponding to the vertices of $\Delta$. In this case, $I_{M}$ is generated solely in degrees 2 and 4:

$$
\begin{aligned}
I_{M}= & \left(p_{1011} p_{1110}-p_{1010} p_{1111}, \quad p_{1001} p_{1100}-p_{1000} p_{1101},\right. \\
& p_{0111} p_{1101}-p_{0101} p_{1111}, \quad p_{0110} p_{1100}-p_{0100} p_{1110}, \\
& p_{0011} p_{0110}-p_{0010} p_{0111}, \quad p_{0011} p_{1001}-p_{0001} p_{1011}, \\
& p_{0001} p_{0100}-p_{0000} p_{0101}, \quad p_{0010} p_{1000}-p_{0000} p_{1010}, \\
& p_{0100} p_{0111} p_{1001} p_{1010}-p_{0101} p_{0110} p_{1000} p_{1011}, \\
& p_{0010} p_{0101} p_{1011} p_{1100}-p_{0011} p_{0100} p_{1010} p_{1101}, \\
& p_{0001} p_{0110} p_{1010} p_{1101}-p_{0010} p_{0101} p_{1001} p_{1110}, \\
& p_{0001} p_{0111} p_{1010} p_{1100}-p_{0011} p_{0101} p_{1000} p_{1110},
\end{aligned}
$$




$$
\begin{aligned}
& p_{0000} p_{0011} p_{1101} p_{1110}-p_{0001} p_{0010} p_{1100} p_{1111}, \\
& p_{0000} p_{0111} p_{1001} p_{1110}-p_{0001} p_{0110} p_{1000} p_{1111}, \\
& p_{0000} p_{0110} p_{1011} p_{1101}-p_{0010} p_{0100} p_{1001} p_{1111}, \\
& \left.p_{0000} p_{0111} p_{1011} p_{1100}-p_{0011} p_{0100} p_{1000} p_{1111}\right) .
\end{aligned}
$$

\section{Toric ideals of hierarchical models}

Hierarchical models generalize the notion of row and column sums of a matrix. For higher dimensional tensors, the models are defined in terms of the facets of some simplicial complex.

\section{Definition 2.1}

(a) Given a $d_{1} \times \cdots \times d_{n}$ table $T \in \bigotimes_{j=1}^{n} \mathbb{R}^{d_{j}}$ and $F \subseteq[n]$, define the $F$-margin of $T$ to be

$$
T_{F}:=\sum_{\left(i_{j} \mid j \notin F\right)} T_{i_{1} \ldots i_{n}} \in \bigotimes_{j \in F} \mathbb{R}^{d_{j}} .
$$

(b) Let $\Delta$ be a simplicial complex on $[n]$, with facets $F_{1}, \ldots, F_{s}$ and $d=$ $\left(d_{1}, \ldots, d_{n}\right)$. Define a linear map $\phi_{\Delta}=\phi_{\Delta, d}: \bigotimes_{i=1}^{n} \mathbb{R}^{d_{i}} \rightarrow \bigoplus_{i=1}^{s}\left(\bigotimes_{j \in F_{i}} \mathbb{R}^{d_{j}}\right)$ sending a table $T$ to $\left(T_{F_{1}}, \ldots, T_{F_{s}}\right) . M(\Delta, d)$ is the matrix representing this map in the standard basis.

Example 2.2 The matrix for the complex with facets $\{1\}$ and $\{2\}$ is

$$
\left[\begin{array}{llll}
1 & 1 & 0 & 0 \\
0 & 0 & 1 & 1 \\
1 & 0 & 1 & 0 \\
0 & 1 & 0 & 1
\end{array}\right] .
$$

The map $\phi: \mathbb{R}^{4} \rightarrow \mathbb{R}^{2} \oplus \mathbb{R}^{2}$, restricted to the probability simplex, sends a $2 \times 2$ table $\left[T_{i j}\right]$ to the 1-dimensional margins obtained by summing over each index: $\left[T_{i j}\right] \mapsto$ $\left(\left[\sum_{j} T_{i j}\right]_{i=1,2},\left[\sum_{i} T_{i j}\right]_{j=1,2}\right)$. These are the row and column sums of the table.

Hierarchical models are a subclass of log-linear models in statistics: sets of all probability distributions whose logarithms are in the linear span of the rows of some matrix $A$. The hierarchical model $\mathcal{M}_{M(\Delta)}$ is the intersection of the toric variety parametrized by the map induced by $M(\Delta)$ and the probability simplex. In the language of algebraic statistics, the facets of the complex determine the sufficient statistics of the model.

In practice, one is interested in performing a random walk on the fibers of the model, defined as the sets of points with the same sufficient statistics. Such random walks are crucial for testing the goodness of fit for the model. The fiber of a point $u$ in the image of $M(\Delta)$, denoted by $\mathcal{F}(u)=\phi_{\Delta}^{-1}(u)$, is a set of all points $v$ such that $M(\Delta) v=M(\Delta) u$. A Markov basis $\mathcal{B} \subseteq \operatorname{ker} M(\Delta)$ for the model $\mathcal{M}_{M(\Delta)}$ is a finite 
set of tensors, called moves, that connects every fiber $\mathcal{F}(u)$ in the following sense: if $T_{1}, T_{2} \in \mathcal{F}(u)$ then $T_{1}=T_{2}+\sum_{i=1}^{k} m_{i}$ and $T_{2}+\sum_{i=0}^{\ell} m_{i} \in \mathcal{F}(u)$ for every $\ell \leq k$, for some collection of moves $\left\{m_{1}, \ldots, m_{k}\right\} \subseteq \mathcal{B}$.

The matrix $M(\Delta)$ of a hierarchical model determines the toric ideal $I_{M(\Delta)}:=$ $\left(x^{u}-x^{v}: u-v \in \operatorname{ker} M(\Delta)\right)$ in the polynomial ring $S:=K\left[x_{1}, \ldots, x_{N}\right]$, where $N$ is the number of entries in the table. A starting point of the field of algebraic statistics is the realization that generators of the ideal form a Markov basis for the model ([7], see also Theorem 1.3.6 in [11]).

Example 2.3 Extending the map for the $2 \times 2$ table from Example 2.2 to complex numbers as follows:

$$
\begin{aligned}
\tilde{\phi}: \mathbb{C}\left[T_{11}, T_{12}, T_{21}, T_{22}\right] & \rightarrow \mathbb{C}\left[r_{1}, r_{2}, c_{1}, c_{2}\right] \\
T_{i j} & \mapsto r_{i} c_{j},
\end{aligned}
$$

we see that the image of $\tilde{\phi}$ restricted to the probability simplex is exactly the image of $\phi$; namely, $T_{i j}$ represents the $(i, j)$-entry in the table. The toric ideal $\operatorname{ker} \tilde{\phi}$ is generated by $T_{11} T_{22}-T_{12} T_{21}$, the determinant of the generic $2 \times 2$ matrix. Therefore, this hierarchical model is the real positive part of the Segre embedding $\mathbb{P}^{1} \times \mathbb{P}^{1} \rightarrow \mathbb{P}^{3}$.

In algebraic statistics, we refer to the degrees of minimal generators of the toric ideal $I_{M(\Delta)}$ as Markov degrees. Surprisingly, we make a link between these Markov degrees and Betti numbers of the monomial ideal $I_{\Delta}$.

\section{Syzygies of Stanley-Reisner ideals}

Here we briefly recall the necessary background and notation. Let $K$ be any field, $R:=K\left[x_{1}, \ldots, x_{n}\right]$ and define $x_{\sigma}:=\prod_{i \in \sigma} x_{i}$ for $\sigma \subseteq[n]=\{1, \ldots, n\}$. Then the Stanley-Reisner ideal of the simplicial complex $\Delta$ is

$$
I_{\Delta}:=\left(x_{\sigma} \mid \sigma \notin \Delta\right) \subseteq R .
$$

There are three basic constructions for a simplicial complex: restrictions, deletions, and links. For $\sigma \subseteq[n]$, the restriction of $\Delta$ to $\sigma$ is defined as $\left.\Delta\right|_{\sigma}:=\{F \in$ $\Delta \mid F \subseteq \sigma\}$. The deletion of $v$ from $\Delta$ is $\Delta_{-v}:=\{F \in \Delta \mid v \notin F\}$. The link of a complex with respect to $F \subseteq[n]$ is defined to be $\operatorname{link}_{\Delta}(F):=\{G \in \Delta \mid G \cap F=\emptyset$, $F \cup G \in \Delta\}$.

The graded Betti numbers $\beta_{i j}$ of $I_{\Delta} \subseteq R$ encode the ranks of the syzygy modules in a minimal free resolution of its coordinate ring $R / I_{\Delta}$ :

$$
0 \rightarrow \bigoplus_{j \in \mathbb{Z}} R(-j)^{\beta_{p j}} \rightarrow \cdots \rightarrow \bigoplus_{j \in \mathbb{Z}} R(-j)^{\beta_{2 j}} \rightarrow \bigoplus_{j \in \mathbb{Z}} R(-j)^{\beta_{1 j}} \rightarrow R \rightarrow R / I \rightarrow 0
$$

Recall that $\beta_{i}\left(R / I_{\Delta}\right):=\sum_{j} \beta_{i j}\left(R / I_{\Delta}\right)$ are the total Betti numbers. If $I_{\Delta}$ and $R$ are understood from the context, we simply write $\beta_{i j}$ for the graded Betti numbers. We can also grade $R$ by $\mathbb{Z}^{n}$ instead of $\mathbb{Z}$ by setting $\operatorname{deg} x_{i}$ to the $i$ th standard unit vector in 
$\mathbb{Z}^{n}$. Then the summands of the $i$ th syzygy module have the form $R(-\mathbf{b})^{\beta_{i \mathbf{b}}}$ for some $\mathbf{b} \in \mathbb{Z}^{n}$. We call $\mathbf{b}$ a multidegree and $\beta_{i \mathbf{b}}$ a multigraded Betti number. Typically, we summarize this numerical data in a standard Macaulay2 [16] Betti diagram, a table whose $(i, j)$ th entry is $\beta_{i, i+j}$ :

$$
\begin{array}{rrr} 
& \cdots & i \\
\text { total: } & \cdots & \beta_{i} \\
\vdots & & \vdots \\
j: & \cdots & \beta_{i, i+j}
\end{array}
$$

This is the notation used in Example 1.1, where, in the interest of readability, we use - in place of 0 .

The fundamental result describing the Betti numbers of Stanley-Reisner ideals is Hochster's formula, which relates the Betti numbers to the simplicial cohomology of the complex.

Theorem 3.1 (Hochster's Formula, [23, Corollary 5.12]) The graded Betti numbers of $R / I_{\Delta}$ are given by

$$
\beta_{i, i+j}\left(R / I_{\Delta}\right)=\sum_{|\sigma|=j} \operatorname{dim}_{K} \widetilde{H}^{j-1}\left(\left.\Delta\right|_{\sigma} ; K\right) .
$$

\section{From Betti diagrams to Markov degrees}

\subsection{Initial degrees}

In the special case of the initial Markov degrees, we can prove a stronger statement which is not true in the general case. The initial degree of a homogeneous ideal $I$, denoted by $\operatorname{init}(I)$, is the smallest degree of a minimal generator of $I$. To state the relationship between $\operatorname{init}\left(I_{\Delta}\right)$ and $\operatorname{init}\left(I_{M(\Delta)}\right)$, we need the following constructions.

In [19], Hoşten and Sullivant observe that $M(\Delta, d)$ is of the following form:

$$
M(\Delta, d)=\left[\begin{array}{ccccc}
A & 0 & 0 & \ldots & 0 \\
0 & A & 0 & \ldots & 0 \\
\vdots & \vdots & \vdots & \ddots & \vdots \\
0 & 0 & 0 & \ldots & A \\
B & B & B & \ldots & B
\end{array}\right]
$$

where there are $d_{1}$ copies of $A$ and $B$. They also note that $A$ and $B$ are matrices corresponding to $\operatorname{link}_{\Delta}(1)$, and to the complex generated by the facets that do not contain the vertex 1 , respectively. Interestingly, even though any $M$ can be built from $A$ and $B$, not every complex can. Instead, an arbitrary complex can be built from links and deletions, so there is a subtle but crucial difference between $\Delta_{-v}$ and the complex defined by $B$. 
Notation From here on, we adopt the following notation: $\Delta$ is a simplicial complex on $n$ vertices, $d=\left(d_{1}, \ldots, d_{n}\right)$ is a sequence of non-negative integers and $M=M(\Delta, d)$. In addition, $A$ and $B$ are the matrices from the above decomposition of $M$. The toric ideal of the model is denoted by $I_{M}$ and lives in the polynomial ring $S$.

It is interesting to note that in some special cases, the matrix of the hierarchical model has a structure that makes an appearance in integer programming. Namely, if $\operatorname{dim} \Delta=d$ and the only facet not containing 1 has dimension $d$, then $B$ is the identity matrix, and $M$ becomes a higher Lawrence lifting of $A$. Then the Graver basis of $I_{M}$ coincides with any minimal Markov basis and any reduced Gröbner basis [26, Theorem 7.1].

Initial degrees of generators of $I_{M}$ and $I_{\Delta}$ are related as follows.

Proposition 4.1 Let $k=\operatorname{init}\left(I_{\Delta}\right)$. Then $\operatorname{init}\left(I_{M}\right)=2^{k-1}$ provided that $d_{i} \geq 2$ for $1 \leq i \leq n$.

Furthermore, let $D=\operatorname{init}\left(I_{M}\right)$. Then, if $\left(u_{1}, \ldots, u_{d_{1}}\right) \in \operatorname{ker} M$ with $u_{i} \in \operatorname{ker} A$ has degree $D$, it is squarefree.

Proof We proceed by induction on $k$. If $k=1$, then there is some vertex not in $\Delta$, and $I_{M}$ contains a linear form. Therefore $\operatorname{init}\left(I_{M}\right)=1$. To see this, without loss of generality, suppose that $\{1\} \notin \Delta$. Then, we may write $M=[B \cdots B]$. Clearly, there is a linear form in $\operatorname{ker} M$. For example, $(1,0, \ldots, 0,-1,0, \ldots, 0) \in \operatorname{ker} M$, where the -1 is in position $\prod_{i=2}^{n} d_{i}+1$.

Suppose $k>1$. Let $v$ be a vertex in the support of a minimal generator of $I_{\Delta}$ with degree $k$. Then, the Stanley-Reisner ideal of $\operatorname{link}_{\Delta}(v)$, regarded as a complex over $[n]-\{v\}$, is $\left(\left(I_{\Delta}: x_{v}\right)+x_{v}\right) /\left(x_{v}\right) \subseteq R /\left(x_{v}\right)$. Due to our choice of $v$, this ideal has initial degree $k-1$. For convenience, we relabel the vertices of $\Delta$ so that $v$ is labeled as vertex 1 , noting that this does not change the initial degrees of $I_{\Delta}$ or $I_{M}$.

By induction, $I_{A}$ has initial degree $2^{k-2}$. Let $u \in \operatorname{ker} A$ have minimal degree. Then $(u,-u, 0, \ldots, 0) \in \operatorname{ker} M$ has degree $2 \cdot 2^{k-2}=2^{k-1}$. We claim that this is the smallest degree of any element of ker $M$. Suppose, to the contrary, that there is some $\left(a_{1}, a_{2}, \ldots, a_{d_{1}}\right) \in \operatorname{ker} M$ with degree less than $2^{k-1}$. Then at least one of the $a_{i}$ 's must have degree less than $2^{k-1} / d_{1} \leq 2^{k-2}$. Now $a_{i} \in \operatorname{ker} A$ provides a contradiction.

To prove the second claim, we may assume that $\operatorname{init}\left(I_{A}\right)=D / 2$. Since the degree of any of the $u_{i}$ 's cannot be smaller than $D / 2$, at most 2 of the $u_{i}$ 's are non-zero: say, $u_{j}=: u$ and $u_{l}=: v$. There are two cases: $\operatorname{deg} u=\operatorname{deg} v=D / 2$, or one of the vectors is 0 while the other has degree $D$. In the first case, each of $u$ and $v$ must be in the Markov basis for $A$. By induction on $n$, both are squarefree and thus the concatenation is also squarefree.

In the second case, since the nonlinear generators of the Stanley-Reisner ideal of the deletion are also minimal generators of the Stanley-Reisner ideal of $\Delta$, we see that having a degree $D$ element in $I_{B}$ forces $\operatorname{init}\left(I_{B}\right)=D$. The claim now follows by induction on the number of vertices. 
Remark 4.2 Results of Proposition 4.1 have been proved in [20, Theorem 5] using only linear algebra, independently and at the same time this manuscript was written.

The only case in which $I_{M}$ has initial degree 1 is when there is some vertex not contained in $\Delta$. In this case, we may simply take quotients to pass to smaller polynomial rings, and thus assume that $\operatorname{init}\left(I_{M}\right) \geq 2$. It then follows from Proposition 4.1 that all degree 2 elements in any Markov basis are squarefree. The squarefree quadratic Markov moves are studied in [17] under the name primitive moves.

\subsection{The general case}

The proof of the main theorem is by induction. We use the following technical result to reduce to smaller complexes.

Lemma 4.3 If $\Delta$ is a complex with $H^{d}(\Delta ; K) \neq 0$ and $H^{d}\left(\Delta_{-v} ; K\right)=0$ for some $v \in \Delta$, then $H^{d-1}\left(\operatorname{link}_{\Delta}(v) ; K\right) \neq 0$.

Proof Define $\operatorname{star}_{\Delta}(v):=\{F \in \Delta \mid\{v\} \cup F \in \Delta\}$. Note that $\operatorname{star}_{\Delta}(v) \cup \Delta_{-v}=\Delta$ and $\operatorname{star}_{\Delta}(v) \cap \Delta_{-v}=\operatorname{link}_{\Delta}(v)$. Applying the Mayer-Vietoris sequence to the above pair we get an exact sequence

$$
\rightarrow H^{i-1}\left(\operatorname{link}_{\Delta}(v)\right) \rightarrow H^{i}(\Delta) \rightarrow H^{i}\left(\operatorname{star}_{\Delta}(v)\right) \oplus H^{i}\left(\Delta_{-v}\right) \rightarrow
$$

Since, by assumption, $H^{d}\left(\Delta_{-v}\right)=0$, and since the star is, by definition, a cone with apex $v$, and thus contactable, the right-most term is 0 . Since the middle term is nonzero, it follows that the left-most term is also non-zero, as required.

The proof of the main theorem will make use of tableaux notation, which can be found, for example, in [11, Chap. 1]. We consider the elements of the Markov basis as binomials in $K\left[p_{b} \mid b \in\left[d_{1}\right] \times \cdots \times\left[d_{n}\right]\right]$ and write the indices as the rows of two matrices. For example, $p_{0111} p_{0001}-p_{0011} p_{0101}$ is written

$$
\left[\begin{array}{llll}
0 & 1 & 1 & 1 \\
0 & 0 & 0 & 1
\end{array}\right]-\left[\begin{array}{llll}
0 & 0 & 1 & 1 \\
0 & 1 & 0 & 1
\end{array}\right] .
$$

Note that, by definition, a tableau is in $I_{M}$ if and only if for every face, $F \in \Delta$, restricting the matrices to only those columns whose indices appear in $F$ gives 0 (that is, the two matrices are equal up to permutations of the rows). In particular, if $i \in \Delta$ and column $i$ of the left-hand matrix is constant, then it must equal the corresponding column of the right-hand matrix. We will make use of this fact in the proof below: we will ignore columns that are constant and pass to $\Delta_{-v}$.

Theorem 4.4 Let $\Delta$ be a complex with $\beta_{i, i+j}\left(R / I_{\Delta}\right) \neq 0$ for some $i$. Then $M(\Delta)$ has a minimal Markov move with degree $2^{j}$.

Proof Choose $i$ to be minimal so that $\beta_{i, i+j}\left(R / I_{\Delta}\right) \neq 0$. Consider the multi-graded minimal free resolution of $R / I_{\Delta}$. There is some $\mathbf{b} \in\{0,1\}^{n}$ with $|\mathbf{b}|=i+j$ 
and $\beta_{i, \mathbf{b}}\left(R / I_{\Delta}\right) \neq 0$. Letting $\sigma:=\operatorname{supp}(\mathbf{b})=\left\{k_{1}, \ldots, k_{i+j}\right\}, H^{j-1}\left(\left.\Delta\right|_{\sigma} ; K\right) \neq 0$ by Hochster's formula. We consider 2 cases depending on the size of $i+j$.

If $i+j<n$, then $\left.\Delta\right|_{\sigma}$ is a proper subcomplex of $\Delta$. By induction on the number of vertices, since the claim is trivial for 1 vertex, $M\left(\left.\Delta\right|_{\sigma}\right)$ has a minimal Markov move $m$ of degree $2^{j}$. Write $m$ in tableaux notation as

$$
m=\left[m_{k_{1}}^{+} \cdots m_{k_{i+j}}^{+}\right]-\left[m_{k_{1}}^{-} \cdots m_{k_{i+j}}^{-}\right]
$$

where each $m_{k}^{ \pm}$is a column vector and the rows are the indices of the variables appearing in the positive and negative parts of the binomial $m$, respectively. Lift $m$ to

$$
\bar{m}=\left[\bar{m}_{1}^{+} \cdots \bar{m}_{n}^{+}\right]-\left[\bar{m}_{1}^{-} \cdots \bar{m}_{n}^{-}\right],
$$

where $\bar{m}_{k}^{+}=\mathbf{0}$ if $k \notin \sigma$ and $\bar{m}_{k_{s}}^{+}=m_{k_{s}}^{+}$otherwise. Clearly $\bar{m} \in I_{M(\Delta)}$ and we need only show that it is minimal. Suppose to the contrary that $\bar{m}$ can be written as

$$
\bar{m}=\sum a_{k} v_{k}
$$

with $a_{k} \in S$ and $v_{k} \in I_{M(\Delta)}$ binomials. Define the support of a binomial $v \in I_{M(\Delta)}$ to be $\operatorname{supp}_{\Delta}(v)=\left\{k \mid v_{k}^{+}, v_{k}^{-}\right.$are not constant $\}$, where $v_{k}^{+}$is the $k$ th column of the tableaux form of $v$, as above. Recall that $\sigma$ is the support of the shift $\mathbf{b}$ in the minimal free resolution. If any of the $v_{k}$ in (1) have $\operatorname{supp}_{\Delta}\left(v_{k}\right) \neq \sigma$, then each monomial in $v_{k}$ must be canceled by some other summand with support $\operatorname{supp}_{\Delta}\left(v_{k}\right)$. Thus we may, without loss of generality, assume that $\operatorname{supp}_{\Delta}\left(v_{k}\right)=\sigma$ for each $v_{k}$ in (1). If $\operatorname{supp}_{\Delta}\left(v_{k}\right)=\sigma$ then $v_{k} \in I_{M\left(\left.\Delta\right|_{\sigma}\right)}$. But then, after deleting the columns not in $\sigma$, we have written $m$ as a linear combination of elements of $I_{M\left(\left.\Delta\right|_{\sigma}\right)}$, contradicting the minimality of $m$. Thus, $\bar{m}$ must be minimal in $I_{M(\Delta)}$. Since $\operatorname{deg} \bar{m}=\operatorname{deg} m=2^{j}$, we are done with this case.

Suppose that $i+j=n$ (and thus $\mathbf{b}=(1,1, \ldots, 1)$ ). By Hochster's formula and the minimality of $i, H^{j-1}(\Delta ; K) \neq 0$ and $H^{j-1}\left(\Delta_{-v} ; K\right)=0$ for every $v \in \Delta$. By Lemma $4.3 H^{j-2}\left(\operatorname{link}_{\Delta}(v) ; K\right) \neq 0$ and thus (using Hochster's formula again) $\beta_{i, i+j-1} R /\left(I_{\operatorname{link}_{\Delta}(v)}+x_{v}\right) \neq 0$. By induction on the number of vertices, $M\left(\operatorname{link}_{\Delta}(v)\right)$ has a minimal Markov move, $m$, of degree $2^{j-1}$. Then, as in the proof of Proposition 4.1 , we can lift $m$ to a minimal Markov move $(m,-m, 0, \ldots, 0)$ with degree $2^{j}$.

Remark 4.5 Note that proof of Theorem 4.4 is, essentially, constructive. Given a non-zero multi-graded Betti-number, $\beta_{i, \mathbf{b}}$, restrict to $\operatorname{supp}(\mathbf{b})$ and then link repeatedly until you arrive at a complex with dimension 0 , at which point the quadratic minimal Markov moves can be explicitly described. Then, lifting the move back to $M(\Delta)$ as in the proof of Theorem 4.4 gives a minimal Markov move for $\Delta$. We will demonstrate this procedure more explicitly in Sect. 5 .

Geometrically, the Betti diagram is determined by the reduced simplicial cohomology of the complex and its sub-complexes via Hochster's formula (see Sect. 3).

Corollary 4.6 If $\widetilde{H}^{j}(\Delta ; K) \neq 0$ for some $j>0$ and some field $K$, then $I_{M}$ has a degree $2^{j+1}$ minimal generator. 
Proof Taking quotients if necessary, we may assume that $f_{0}(\Delta)=n$. Hochster's formula says that if we have a vector $a \in\{0,1\}^{n}$ and $A=\operatorname{supp}(a)$, then we can compute the $\mathbb{Z}^{n}$-graded Betti numbers by

$$
\operatorname{dim} \widetilde{H}^{|A|-j-1}\left(\left.\Delta\right|_{A} ; K\right)=\beta_{j, a}^{K}\left(R / I_{\Delta}\right) .
$$

Let $A=[n]$, the only set such that $\left.\Delta\right|_{A}=\Delta$. Since all the Betti numbers of $I_{\Delta}$ are squarefree and $(1, \ldots, 1)$ is the only squarefree integer vector with sum $n$, we get $0 \neq \operatorname{dim} \widetilde{H}^{j}(\Delta ; K)=\beta_{n-j-1, n}\left(R / I_{\Delta}\right)$. Therefore, the Betti diagram has a non-zero entry in row $n-(n-j-1)=j+1$.

Example 4.7 Corollary 4.6 can be applied to all connected graphs. For example, if $\Delta$ is a cycle, or any graph containing a cycle as a vertex-induced subgraph, then $I_{M}$ must have a degree 4 element in its Markov basis, because $\Delta$ has non-zero first cohomology.

Similarly, if $\Delta$ is a simplicial $k$-sphere, then $H^{k}(\Delta ; K) \cong K$. Hence the Markov basis must contain a degree $2^{k+1}$ element. For example, any Markov basis of a hierarchical model associated to an octahedron contains at least one move with degree 8 .

Remark 4.8 For graphs, it has recently been shown in [22] that the hierarchical model is generated in degrees 2 and 4 if and only if the graph has no $K_{4}$-minors. If $\operatorname{dim} \Delta=1$ then, using Hochster's formula, $\beta_{i, i+j} R / I_{\Delta}=0$ whenever $j>2$, so our theorem can only predict generators in degrees 2 and 4 . If $\Delta$ has a $K_{4}$-minor, then the dimension of $\widetilde{H}^{1}(\Delta ; K)$ is at least that of $\widetilde{H}^{1}\left(K_{4} ; K\right)$, which is three. Assuming that $\Delta$ is connected, this means our theorem can give an exact listing of generators only if the right-most entry in the Betti diagram is at most two. This fails in dimension two.

\subsection{Linear resolutions}

Theorem 4.4 can be used to obtain information about the Stanley-Reisner ideal from the Markov basis. For example, if the Markov basis contains elements of only three distinct degrees, then the Betti diagram of $I_{\Delta}$ can contain at most three non-zero rows. In fact, it is likely that there will be fewer than three, since Theorem 4.4 only includes the Markov basis elements whose degree is a power of 2 . As a special case, the result provides a class of complexes whose Stanley-Reisner ideals have linear resolutions.

Corollary 4.9 If $I_{M}$ is generated in a single degree, then $I_{\Delta}$ has a linear resolution over every field.

Decomposable complexes are clique complexes of chordal graphs. Theorem 4.4 in [15] characterizes decomposable models as those whose toric ideals are generated in degree 2. This is one of the few classes of models where the generators are known. With that in mind, we obtain the following:

Corollary 4.10 If $\Delta$ is decomposable then $I_{\Delta}$ has a 2-linear resolution over every field. 
This recovers a theorem of Fröberg. Namely, clique complexes of chordal graphs are examples of flag complexes. In [14], Fröberg characterized the Stanley-Reisner rings of flag complexes that have a linear resolution: they are exactly clique complexes of chordal graphs. His proof uses Mayer-Vietoris sequences and does not give a combinatorial formula for the Betti numbers. Recently, an easier proof of this proposition, along with a formula for the Betti numbers, appeared in [10, Theorem 3.2].

\section{Constructing the moves}

In the previous section, we showed that the Betti diagram of the Stanley-Reisner ideal implies the existence of toric ideal generators of certain degrees. Here we give a way to construct those binomials explicitly. In general, this is a non-trivial task for any reasonably complicated model. We focus on binary hierarchical models, that is, on the case when all random variables have two states. The general result follows similarly.

For a general recipe, we introduce notation to describe the columns of the tableaux that we can construct using the procedure of the theorem. First, we define the alternating vectors $\varepsilon_{k}^{\ell}$ by

$$
\begin{aligned}
\varepsilon_{k}^{1} & =[\underbrace{0 \cdots 0}_{k \text { times }} \overbrace{1 \cdots 1}^{k \text { times }}]^{T}, \\
\varepsilon_{k}^{\ell} & =[\underbrace{\varepsilon_{k}^{1} \cdots \varepsilon_{k}^{1}}_{\ell \text { times }}]^{T} \\
& =\left[\begin{array}{ll}
\varepsilon_{k}^{\ell-1} & \varepsilon_{k}^{1}
\end{array}\right]^{T},
\end{aligned}
$$

so that $\varepsilon_{2}^{1}=\left[\begin{array}{llll}0 & 0 & 1 & 1\end{array}\right]^{T}$ while $\varepsilon_{1}^{2}=\left[\begin{array}{llll}0 & 1 & 0 & 1\end{array}\right]^{T}$. In general, $\varepsilon_{k}^{\ell}$ has alternating blocks of 0 's and 1's; $k$ is the length of each block and $\ell$ is the number of blocks.

Additionally, we define column vectors $\alpha_{i}$ by $\alpha_{1}=[0]$ and $\alpha_{i}=\left[\alpha_{i-1} \overline{\alpha_{i-1}}\right]^{T}$, where $\bar{\alpha}$ is the binary complement of $\alpha$, defined by $\bar{\alpha}_{i}:=1-\alpha_{i}$. Here, $\alpha_{1}=\left[\begin{array}{ll}0 & 1\end{array}\right]^{T}$,

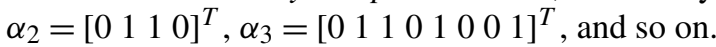

Proposition 5.1 Suppose that $j$ is minimal such that $\beta_{i, i+j}\left(R / I_{\Delta}\right) \neq 0$ and $\mathbf{b} \in$ $\{0,1\}^{n}$ such that $\beta_{i, \mathbf{b}}$ with $|\mathbf{b}|=i+j=d$. If $\operatorname{supp}(\mathbf{b})=\left\{i_{1}, \ldots, i_{d}\right\}$ with $i_{1}<i_{2}<$ $\cdots<i_{d}$, then $[u]-[v]$ is a minimal generator of $I_{M}$ with degree $2^{d-1}$ if, for $j<d$, the $i_{j}$ th columns of $u$ and $v$ are $\varepsilon_{2^{d-j}}^{2^{j-1}}$, and the $i_{d}$ th columns of $u$ and $v$ are $\alpha_{d}$ and $\overline{\alpha_{d}}$, respectively. The other columns of $u$ and $v$ are equal to each other and each is either $[0 \cdots 0]^{T}$ or $[1 \cdots 1]^{T}$. There are a total of $2^{n-d}$ such moves for each degree $d$ minimal generator of $I_{\Delta}$.

Proof We induct on $n$. If $n=1$ then there is only one complex with a non-trivial Stanley-Reisner ideal, $\Delta=\{\emptyset\}$. One easily sees that the single binomial [0] $-[1]$ forms a minimal Markov basis. 
Suppose that $n>1$. By permuting the vertices of $\Delta$, we may, without loss of generality, assume that $\mathbf{b}=(1,1, \ldots, 1,0, \ldots, 0)$. If $i+j<n$ then, as in the proof

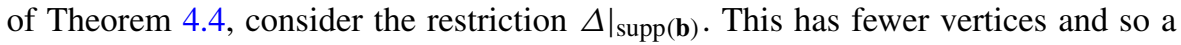
minimal Markov move $m$ of the claimed form exists by induction. Then, using the notation of the proof of Theorem 4.4, $m$ can be lifted to a minimal Markov move $\bar{m}$ of $I_{M}$.

Now suppose that $i+j=n$ so that $\mathbf{b}=(1,1, \ldots, 1)$ and consider $\operatorname{link}_{\Delta}(1)$. Let $\left[u^{\prime}\right]-\left[v^{\prime}\right]$ be the claimed minimal Markov move for $M\left(\operatorname{link}_{\Delta}(1)\right)($ which, as before, exists by induction). Then

$$
\left[\begin{array}{cc}
0 & u^{\prime} \\
1 & v^{\prime}
\end{array}\right]-\left[\begin{array}{ll}
0 & v^{\prime} \\
1 & u^{\prime}
\end{array}\right]
$$

is a minimal generator of $I_{M}$. By construction, the first column of this matrix is $\varepsilon_{2^{d-1}}^{1}$, the $d$ th column is $\alpha_{d}$, while column $j$, for $2 \leq j \leq d-1$, is $\varepsilon_{2^{d-j}}^{2^{j}}=\left[\varepsilon_{2^{d-j}}^{2^{j-1}} \varepsilon_{2^{d-j}}^{2^{j-1}}\right]^{T}$.

Example 5.2 We can construct some quadratic Markov moves for the complex with facets

$$
\{\{12\},\{13\},\{14\},\{15\},\{23\},\{24\},\{25\},\{34\}\} .
$$

This has Stanley-Reisner ideal $\left(x_{4} x_{5}, x_{3} x_{5}, x_{1} x_{2} x_{5}, x_{1} x_{3} x_{4}, x_{2} x_{3} x_{4}, x_{1} x_{2} x_{4}, x_{1} x_{2} x_{3}\right)$ and Betti diagram

$\begin{array}{rrrrr} & 0 & 1 & 2 & 3 \\ \text { total: } & 1 & 7 & 10 & 4 \\ 0: & 1 & . & . & . \\ 1: & . & 2 & 1 & . \\ 2: & . & 5 & 9 & 4\end{array}$

The minimal entries in row 1 are the two quadratic minimal generators of $I_{\Delta}, x_{3} x_{5}$ and $x_{4} x_{5}$. First, consider $x_{3} x_{5}$ so that $\mathbf{b}=(0,0,1,0,1)$. Then Proposition 5.1 shows that

$$
\left[\begin{array}{lllll}
0 & 0 & 0 & 0 & 0 \\
0 & 0 & 1 & 0 & 1
\end{array}\right]-\left[\begin{array}{lllll}
0 & 0 & 0 & 0 & 1 \\
0 & 0 & 1 & 0 & 0
\end{array}\right]
$$

is a minimal generator of $I_{M}$. By altering this construction slightly, any of the first, second or fourth columns could be 1 instead of 0 (but must be the same on both sides), giving us a total of eight minimal generators. Starting with $x_{4} x_{5}$ and following the same procedure gives us the minimal Markov move

$$
\left[\begin{array}{lllll}
0 & 0 & 0 & 0 & 0 \\
0 & 0 & 0 & 1 & 1
\end{array}\right]-\left[\begin{array}{lllll}
0 & 0 & 0 & 0 & 1 \\
0 & 0 & 0 & 1 & 0
\end{array}\right] .
$$

As before, the first, second and third columns can contain 1's instead of 0's, giving us 8 more quadratic generators, for a total of 16 . On the other hand, computing the Markov basis with 4 ti 2 gives the result that there are 24 quadratic moves in total. We will address this discrepancy shortly.

Now, we construct a degree 4 minimal Markov move from the generator $x_{1} x_{3} x_{4}$.

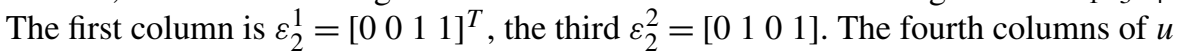


and $v$ are $\alpha_{2}=\left[\begin{array}{llll}0 & 1 & 1 & 0\end{array}\right]^{T}$ and $\overline{\alpha_{2}}=\left[\begin{array}{llll}1 & 0 & 0 & 1\end{array}\right]^{T}$. The other columns we may fill with 0 's or 1 's as we like. We get

$$
\left[\begin{array}{lllll}
0 & 0 & 0 & 0 & 0 \\
0 & 0 & 1 & 0 & 1 \\
1 & 0 & 0 & 0 & 1 \\
1 & 0 & 1 & 0 & 0
\end{array}\right]-\left[\begin{array}{lllll}
0 & 0 & 0 & 0 & 1 \\
0 & 0 & 1 & 0 & 0 \\
1 & 0 & 0 & 0 & 0 \\
1 & 0 & 1 & 0 & 1
\end{array}\right],
$$

along with three other binomials obtained by varying columns 2 and 4 . Iterating over all the cubic generators of $I_{\Delta}$ we construct a total of 20 degree 4 binomials, out of the 520 that a minimal Markov basis contains.

Remark 5.3 Each of the moves $[u]-[v]$ that Proposition 5.1 constructs from a degree $d$ monomial has the property that all of the columns of $u$ and $v$, except one, are equal, and all but $d$ of the columns are either all 0's or all 1's. However, not every degree $2^{d-1}$ minimal Markov move can be constructed in this way. For the complex

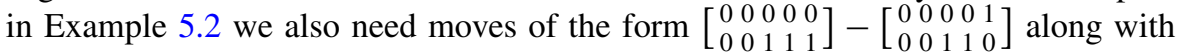
three other moves obtained by replacing either of the first two columns with $\left[\begin{array}{ll}1 & 1\end{array}\right]^{T}$ on both sides. The other four (which, together with the 16 from Example 5.2 give us all

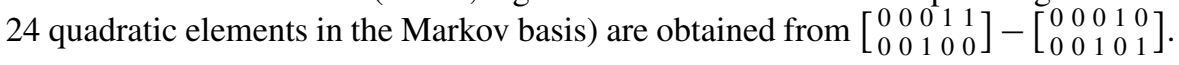
To study these, one may want to look further out in the resolution of $I_{\Delta}$.

Note that the lone linear syzygy of the 2 quadratic generators has multidegree $(0,0,1,1,1)$, suggesting that this is the source of the quadratic binomials missed by Proposition 5.1. Even in the smallest degrees, the minimal generators alone of $I_{\Delta}$ do not give enough information to construct all of the minimal Markov moves.

\section{Unpredictable moves and open problems}

The following example shows that the converse of Theorem 4.4 does not hold.

Example 6.1 Contrast Example 1.1 with the complex whose Stanley-Reisner ideal is

$$
I=\left(x_{3} x_{4} x_{5}, x_{1} x_{2} x_{5}, x_{1} x_{4} x_{5}, x_{1} x_{2} x_{3}\right),
$$

the Alexander dual of a 5-path. The Betti diagram, over any field, is

$\begin{array}{rccc} & 0 & 1 & 2 \\ \text { total: } & 1 & 4 & 3 \\ 0: & 1 & . & . \\ 1: & . & . & . \\ 2: & . & 4 & 3\end{array}$

Notice that only a degree 4 generator is predicted for $I_{M}$. Yet the binary model over this complex has generators in degrees 4, 6, 8, 10 and 12 .

Considering Example 6.1 and Corollary 4.9, we pose a natural question: 
Problem 6.2 Among those complexes with linear resolutions over every field, which have a Markov basis concentrated in a single degree? In particular, are there any necessary or sufficient conditions on the Betti numbers (equivalently, on the $f$-vector of $\Delta$ ) to have a Markov basis in a single degree?

In the special case where $\Delta$ is a graph, the answer to Problem 6.2 is known: for connected graphs, $M(\Delta)$ has a quadratic Markov basis if and only if $\Delta$ is a tree, which is true if and only if $I_{\Delta}$ has linear minimal free resolution, or if and only if $f(\Delta)=(1, n, n-1)$. As Example 6.1 shows, this fails for higher dimensions.

The construction behind our main Theorem does produce all the quadratic moves for all complexes. However, we do not know if restricting the family of complexes will allow this procedure to produce all moves in other degrees:

Problem 6.3 Are there any complexes where the Markov moves we construct give all the moves of that degree?

In fact, if the family of complexes is restricted, it would be interesting to see if there is a similar but more tailored construction which will produce more Markov moves then we do at the moment.

The reader will note that our proof only looks at the first terms in each row of the Betti diagram of $I_{\Delta}$. It is natural to expect that more can be said about the toric ideal $I_{M}$ if one considers the rest of the Betti diagram. In particular, we may ask the following:

Problem 6.4 Since we have seen that the regularity of $I_{\Delta}$ predicts something about the generators of $I_{M}$, what can be said about the projective dimension? For example, does the length of each row in the Betti diagram of the Stanley-Reisner ideal predict the number of toric Markov moves of the degree predicted by that non-zero row?

A different way to think about the proof of the main Theorem 4.4 is that we are, essentially, linking the complex $\Delta$ repeatedly. This, in turn, gives a series of filtrations, through which we trace the Markov moves by tracing the inclusion maps. We have not tried to obtain different kinds of Markov moves using different filtrations. To that end, we propose the following problem:

Problem 6.5 Can additional information on the (generators of) the ideal $I_{M}$ be captured by examining the homology of various filtrations of $\Delta$ ?

The method of the proof for Theorem 4.4 does work for all models, but provides best results for binary models only. It is not unreasonable to ask for a better bound for non-binary models. For example:

Problem 6.6 How can one obtain a better bound for models whose vertices have more than two states? In particular, is there a way to change the grading on $I_{\Delta}$ so as to mimic the proof of Theorem 4.4, but provide more information about the Markov degrees? 
Interestingly, the Betti-row bound from Theorem 4.4, combined with the generalized toric fiber product construction, gives evidence in support of Conjecture 6.6 of [12], which proposes that the degrees of the Markov moves for the binary model on a triangulation of an $n$-dimensional sphere are at most $2^{n+1}$.

We conclude this note by a computational answer to a natural question motivated by Example 5.2 and Remark 5.3: how much do the predictions offered by Theorem 4.4 differ from the actual Markov bases.

The table below summarizes the results of computer experiments in which we used 4 ti 2 to compute the Markov bases and compared them to the resolutions obtained using Macaulay2. All resolutions were computed over the rationals. The rows in the table give the number of vertices in the complex, the columns represent the number of degrees not predicted. The $(i, j)$-entry in the table is the number of complexes on $i$ vertices for which $j$ Markov degrees are not predicted by our results.

\begin{tabular}{lrrrrrrrr}
\hline$n$ & \multicolumn{8}{c}{ Number of degrees not predicted } \\
\cline { 2 - 8 } & 0 & 1 & 2 & 3 & 4 & 5 & 6 & 7 \\
\hline 3 & 18 & 1 & & & & & & \\
4 & 44 & 8 & & & & & & \\
5 & 17 & 3 & 9 & 24 & 8 & & & \\
6 & 33 & 4 & 1 & 5 & & 2 & 2 \\
7 & 1 & & & & & & & \\
8 & 1 & & & & & & & \\
Total & 114 & 16 & 10 & 29 & 8 & & 2 & 2 \\
\hline
\end{tabular}

We have also computed all complexes on four vertices. Of these, only two complexes contain generators in degrees not predicted by Theorem 4.4. Interestingly, the first is the complete graph $K_{4}$, which, by [22], must have a generator with degree larger than 4 . The second is homotopy equivalent, as a topological space, to $K_{4}$, and thus has a similar Betti diagram.

Acknowledgements This project started while the authors attended the 2007 IMA summer program for graduate students on Applicable Algebraic Geometry held at Texas A\&M University, and was continued in part during the Algebraic Statistical Models Workshop at SAMSI in January 2009. The authors would like to thank Alexander Engström, Thomas Kahle, Uwe Nagel, and Seth Sullivant for helpful references, discussions and comments on a much earlier version of this manuscript. We are also grateful to the anonymous referees for the careful reading of the paper and suggested improvements.

\section{References}

1. 4ti2 team: 4ti2 - a software package for algebraic, geometric and combinatorial problems on linear spaces. Available at www.4ti2.de

2. Aoki, S., Takemura, A.: Minimal bases for a connected Markov chain over $3 \times 3 \times k$ contingency tables with fixed two-dimensional marginals. Aust. N. Z. J. Stat. 45(2), 229-249 (2003)

3. Aoki, S., Takemura, A.: The largest group of invariance for Markov bases and toric ideals. J. Symb. Comput. 43(5), 342-358 (2008) 
4. Bruns, W., Herzog, J.: Cohen-Macaulay Rings. Cambridge University Press, Cambridge (1993). Revised edn.

5. Cox, D., Little, J., O’Shea, D.: Using Algebraic Geometry. Graduate Texts in Mathematics, vol. 185. Springer, Berlin (2005)

6. De Loera, J.A., Onn, S.: Markov bases of three-way tables are arbitrarily complicated. J. Symb. Comput. 41(2), 173-181 (2006)

7. Diaconis, P., Sturmfels, B.: Algebraic algorithms for sampling from conditional distributions. Ann. Stat. 26(1), 363-397 (1998)

8. Dobra, A.: Markov bases for decomposable graphical models. Bernoulli 9(6), 1-16 (2003)

9. Dobra, A., Fienberg, S.E.: Bounds for cell entries in contingency tables given marginal totals and decomposable graphs. Proc. Natl. Acad. Sci. USA 97(22), 11885-11892 (2000) (electronic)

10. Dochtermann, A., Engström, A.: Algebraic properties of edge ideals via combinatorial topology. The Electronic Journal of Combinatorics 16(2) (2009)

11. Drton, M., Sturmfels, B., Sullivant, S.: Lectures on Algebraic Statistics. Oberwolfach Seminars, vol. 39. Birkhäuser, Basel (2009)

12. Engström, A., Kahle, T., Sullivant, S.: Multigraded commutative algebra of graph decompositions, submitted. arXiv.org:1102.2601

13. Fienberg, S.E.: The Analysis of Cross-Classified Categorical Data, 2nd edn. The MIT Press, Cambridge (1980)

14. Fröberg, R.: On Stanley-Reisner rings. In: Topics in Algebra, part 2. Banach Center Publ., vol. 26, pp. 57-70. PWN, Warsaw (1990)

15. Geiger, D., Meek, C., Sturmfels, B.: On the toric algebra of graphical models. Ann. Stat. 34(3), 14631492 (2006)

16. Grayson, D.R., Stillman, M.E.: Macaulay 2, a software system for research in algebraic geometry. Available at http://www.math.uiuc.edu/Macaulay2/

17. Hara, H., Aoki, S., Takemura, A.: Minimal and minimal invariant Markov bases of decomposable models for contingency tables. Bernoulli 16(1), 208-233 (2010)

18. Hara, H., Takemura, A., Yoshida, R.: Markov bases for two-way subtable sum problems. J. Pure Appl. Algebra 213(8), 1507-1521 (2009)

19. Hoşten, S., Sullivant, S.: A finiteness theorem for Markov bases of hierarchical models. J. Comb. Theory, Ser. A 114(2), 311-321 (2007)

20. Kahle, T.: Neighborliness of marginal polytopes. Beitrage Algebra Geom. 51(1), 45-56 (2010)

21. Kahle, T.: On boundaries of statistical models. Ph.D. thesis at University of Leipzig (2010)

22. Král, D., Norine, S., Pangrác, O.: Markov bases of binary graph models of $K_{4}$-minor free graphs. J. Comb. Theory, Ser. A 759-765 (2010)

23. Miller, E., Sturmfels, B.: Combinatorial Commutative Algebra. Springer, Berlin (2005)

24. Pachter, L., Sturmfels, B.: Algebraic Statistics for Computational Biology. Cambridge University Press, Cambridge (2005)

25. Pistone, G., Riccomagno, E., Wynn, H.: Algebraic Statistics. CRC, Boca Raton (2000)

26. Sturmfels, B.: Gröbner Bases and Convex Polytopes. University Lecture Series, vol. 8. American Mathematical Society, Providence (1996)

27. Sullivant, S.: Toric fiber products. J. Algebra 316(2), 560-577 (2007) 